\title{
Ranking of Teaching Evaluation Index System of Public Physical Education Curriculum Based on Management by Objectives Using Analytic Hierarchy Process
}

\author{
Chenyang Zhao' ${ }^{1}$ and A. Gudamu (D) $^{2}$ \\ ${ }^{1}$ Wuxi Vocational Institute of Arts \& Technology, Wuxi, Jiangsu 214222, China \\ ${ }^{2}$ Sungkyunkwan University, Department of Social Welfare, Jongno-gu, Myeongnyun-dong 3-ga 53, Seoul 110-745, Republic \\ of Korea \\ Correspondence should be addressed to A. Gudamu; 06122612@cumt.edu.cn
}

Received 22 June 2021; Revised 24 August 2021; Accepted 11 September 2021; Published 24 September 2021

Academic Editor: Fazlullah Khan

Copyright (c) 2021 Chenyang Zhao and A. Gudamu. This is an open access article distributed under the Creative Commons Attribution License, which permits unrestricted use, distribution, and reproduction in any medium, provided the original work is properly cited.

\begin{abstract}
Teaching evaluation is an important means to ensure the teaching quality of public physical education in colleges and universities. At present, physical education in colleges and universities is constantly changing, and the evaluation system of public physical education has also changed greatly. Based on the perspective of management by objectives, this paper makes a comprehensive evaluation of physical education teaching from two aspects: teachers' teaching preparation and practical teaching. It takes the cultivation of students' sports habits and lifelong sports consciousness as the ultimate goal, establishes 22 indexes to form an index system, and follows the requirements of the new curriculum teaching reform for the development of physical education. In order to continuously improve the level of physical education teaching as the guiding concept, the indexes are refined and decomposed. To determine the weight score of the three-level indexes and the index weight ranking, AHP is used, which is a formal method used to derive ranking from pairwise comparison technique, in accordance with a certain logical relationship, and provide reference for the evaluation of public physical education teaching in colleges and universities. In order to achieve the multiple objectives of public physical education curriculum as the starting point, attention should paid to the teaching focus of public physical education curriculum, and a comprehensive reform strategy should be put forward.
\end{abstract}

\section{Introduction}

Management by objectives is known as "management in management" and widely used in various fields. The concept of management by objectives was put forward by Peter Drucker in 1954. Its basic idea is that the organization first formulates the general goal in a certain period of time and then designates the goals of each department and individual according to the general goal. At the same time, it carries out the implementation of the plan and carries out regular evaluation, summary, and feedback. Finally, it makes improvement according to the existing problems, which is mainly a kind of management according to the set goals. In this paper, a new method is proposed [1]; if there is no clear goal, many areas of work may be ignored, which will directly lead to the conclusion that the "goal cannot be completed." Therefore, colleges and universities are ultimately managed according to the objectives, which is also the most basic principle in the management process.

Management by objectives (MBO) is a scientific and modern management mode, which includes three stages, namely, goal setting stage, goal realization stage, and achievement evaluation stage. $\mathrm{MBO}$ is a kind of management system that has been widely valued by countries all over the world since 1980s. Although the definition and specific implementation methods of $\mathrm{MBO}$ are different at home and abroad, the essence of $\mathrm{MBO}$ is to emphasize management according to objectives, that is, to carry out a series of management activities around the determination of objectives and the realization of objectives. The main 
characteristics of this kind of management are as follows: first, it emphasizes the purpose of activities and the setting of future research and objective system; second, it emphasizes the use of objectives to unify and guide the thoughts and actions of all personnel, so as to ensure the integrity of the organization and the consistency of actions; third, it emphasizes the systematic and overall management according to objectives, so as to make the management process, personnel, methods, and work arrangements revolve around objectives; fourth, it gives full play to people's enthusiasm, initiation, and creativity, implementing self-management and self-control according to the requirements of objectives, so as to improve the ability to adapt to environmental changes; fifth, it emphasizes the evaluation of management performance according to the results of objectives, so as to ensure the satisfactory results of management activities [2].

The function of colleges and universities is to adhere to the socialist direction of running a school, fully implement the party's educational policy, and do a good job in personnel training, scientific research, and social services in accordance with the objective laws of higher education and teaching. The introduction of management by objectives in colleges and universities is to set the general objectives of the above three functions of colleges and universities, by which the teaching units, functional departments, and all teachers and students can be guided to formulate their own subobjectives and individual objectives, and accordingly determine the action plan, organize the implementation, and conduct regular examination and feedback. Specifically, the meaning of management by objectives in colleges and universities includes four aspects: firstly, the management personnel and teaching staff of the teaching unit of the administrative department of the party committee in the university jointly agree on the general objectives of the university on the basis of goal orientation [3]; secondly, the general objectives are decomposed into tasks, responsibilities, and subobjectives to be completed by each unit, department, and person; then, taking the general goal and subgoal as the basis for each unit, department, and individual to carry out their work, all activities of the school are centered around achieving these goals, which organically combines the performance of duties with the realization of goals; finally, the measurement and evaluation of each department and individual are carried out on the basis of goals, and the results are fed back to urge each unit, department, and individual to continuously improve their work [4].

In recent years, with the deepening development of school physical education reform in China, the proposal of various physical education ideas, and the reform of curriculum structure, great changes have taken place in college physical education. It is difficult for the original evaluation system of physical education to adapt to the requirements of the new situation. As for sports themselves, their most essential function is being an effective means of fitness. Without their most essential function, sports will lose their own significance. Due to the diversification of school physical education teaching objectives, as well as the influence of happy sports and weakening sports skills teaching ideas, physical education has become a show class to a certain extent, and the amount and intensity of physical exercise have not reached the standards and requirements to a certain extent, which has not played a good role in strengthening students' physique and cultivating lifelong sports habits and awareness. The decline of students' physique is affected by many factors, but the deviation of the evaluation standard of physical education is the most direct and important reason. Traditionally, there are many problems, such as the serious lack of effectiveness, subjectivity, creativity, and emotion. Physical education should return to its origin; in addition to completing the task of strengthening students' physique, it should also take the cultivation of students' sports habits and lifelong sports consciousness as the ultimate goal. Nowadays, the curriculum reform and school physical education work is becoming more and more important and urgent. It is of great significance to study and determine the evaluation standard system of school's physical education teaching for improving the quality of school physical education teaching.

Teaching evaluation refers to the use of scientific and feasible evaluation methods to evaluate the value of teaching process and teaching results, so as to provide reliable information and scientific basis for improving teaching and teaching quality [5]. In college physical education, scientific and reasonable teaching evaluation of public physical education is an important part of improving the overall teaching level and achieving the goal of physical education.

Public physical education is an important part of college curriculum and teaching management. Improving the teaching quality of public physical education is not only related to the evaluation of college education, but also particularly important to improve the physical quality and physical and mental health of students. With the change of educational environment and students' multilevel demand for physical education [6], the traditional teaching method of public physical education has been unable to meet the main needs of students and the needs of social development, so the teaching evaluation of public physical education should also adapt to this change and should be advanced. The following contributions are made in this regard by conducting this study.

(i) Constructing a teaching evaluation index system based on 22 criteria.

(ii) Formulating a comprehensive reform strategy of physical education curriculum teaching and improvement of teaching quality.

(iii) Conducting a quantitative study on physical education curriculum using expert judgment, for the target evaluation.

(iv) Using analytic hierarchy process (AHP) as a decision making tool combined with public physical education curriculum.

The rest of the paper is organized according to the following pattern. In Section 2, the development of a teaching evaluation index system of public physical education curriculum based on management by objectives is discussed. Section 3 proposes a reform strategy in public physical education curriculum, followed by conclusion. 


\section{Research on Teaching Evaluation Index System of Public Physical Education Curriculum Based on Target Management}

2.1. Design Principles of Teaching Evaluation Index System. The design of teaching evaluation index system needs to follow three principles: The first is goal orientation. Since it is management by objectives, the index system and its evaluation should be closely linked with the target tasks undertaken by all units and departments. For the indexes with target tasks, the completion of the target tasks should be the most important evaluation basis. The second is focus on the work. In the teaching evaluation index system, the school's key work and related objectives should occupy a larger weight, highlighting the evaluation of the school's key work. The third is to pay attention to the implementation of the system. For some tasks and goals that are difficult to quantify but require constant struggle, the main basis for assessment should be in accordance with the school's norms and regulations. The setting of evaluation index should be combined with the actual work of teaching process management [7]. The evaluation index should cover all the work contents of teaching process, which requires relevant functional departments to classify and refine the work of teaching process management. This process is similar to the process of target decomposition, but the difference is that the setting of evaluation index pays more attention to the completion quality standard of specific evaluation points and defines the corresponding score of different quality standards. The score of evaluation should be determined according to the importance of evaluation indexes, the difficulty of completion, the intensity of work content, and other factors. For quantitative indexes, we can set a certain data formula to convert the completion into the corresponding specific score. For the indexes that cannot be quantified, we can only make a comprehensive evaluation based on the collection and arrangement of usual work materials.

2.2. Construction of Teaching Evaluation Index System of Public Physical Education. As far as the teaching process management of physical education curriculum in colleges and universities is concerned, the target evaluation index system is mainly formulated for each teaching college and department, so as to bring the target management work into the scientific and standardized management evaluation system [8], which is convenient for the implementation and evaluation of the target, and realize the efficiency of target management. All the management work of the school, such as theoretical teaching, practical teaching, teaching reform and construction, experimental base construction, and examination work, can be attributed to the responsibilities of each teaching department, and its specific tasks need to be implemented and completed by each department. Therefore, the department in charge of teaching in colleges and universities must establish different and perfect teaching evaluation index system.

\subsubsection{Establishment of Evaluation Index System}

(1) Determining the Evaluation Object and Target. According to the purpose of the study, the evaluation object of this paper is the public physical education curriculum in colleges and universities. The objective of evaluation is to make a comprehensive evaluation of physical education teachers, students, and physical education teaching process.

(2) Determining the Evaluation Index System. For the evaluation of public physical education teaching quality, this paper mainly researches the physical education teachers themselves, physical education, student activities, and other aspects and applies target management point of view on physical education teaching comprehensive research. Physical education teaching quality is not limited to a classroom teaching evaluation and investigation; it should integrate the whole teaching process of a semester or academic year, and students' extracurricular sports activities should also be included in sports teaching evaluation [9]. In order to meet the needs of lifelong education, lifelong learning, and lifelong development, the contents of the evaluation index system are determined. Considering the multiple initiatives of the evaluation, the learners' knowledge ability, skills, and other aspects through learning and the developmental evaluation are highlighted. Through the methods of expert research and questionnaire survey, the establishment of the index system is determined; through the process of primary selection index, expert evaluation (primary screening), and statistical optimization (secondary screening), a total of 22 evaluation indexes are determined to constitute the evaluation index system, and the results are shown in Table 1.

\subsubsection{Determining the Weight of Evaluation Index}

(1) Construction of Judgment Matrix. After defining the hierarchical structure and determining the index system, the next step is for the experts to judge and compare the factors of the criterion layer and the index layer and construct the judgment matrix.

Suppose that the weight of matrix $A$ is $a_{k}$; there are four indexes (criteria) associated with $A_{k}$, namely, $B_{1}, B_{2}, B_{3}$, and $B_{4}$; and their weights are set as $b_{1}, b_{2}, b_{3}$, and $b_{4}$, respectively.

Firstly, through comparison, experts get the ratio of the relative importance of index (or criterion) $B_{i}$ to that of index (or criterion) $B_{j}$, namely, $b_{i j}$. The specific operation method is as follows:

If $B_{i}$ and $B_{j}$ are equally important, then $b_{i j}$ is 1 and $b_{j i}$ is 1 ; if $B_{i}$ is more important than $B_{j}$, then $b_{i j}$ is 5 and $b_{j i}$ is $1 / 5$; if $B_{i}$ is more important than $B_{j}$, then $b_{i j}$ is 9 and $b_{j i}$ is $1 / 9$. According to the value of $b$, a judgment matrix can be determined.

The judgment matrix is constructed layer by layer. However, since different experts may have different understanding, there is often some bias. In order to make the final evaluation reasonable and scientific, multiple experts are required to participate. That is to say, for the same index, 
TABLe 1: Teaching evaluation index system: three-level index construction.

\begin{tabular}{|c|c|c|}
\hline Level 1 indexes & Level 2 indexes & Level 3 indexes \\
\hline \multirow{5}{*}{$\begin{array}{l}\text { Teacher's preparation for } \\
\text { teaching, A }\end{array}$} & Inspection of site facilities, A1 & $\begin{array}{c}\text { Site safety inspection, A1.1 } \\
\text { The number of teaching facilities required is adequate, A1.2 } \\
\text { Talking to students in and out of class, A2.1 }\end{array}$ \\
\hline & Mastery of exercise level, A2 & $\begin{array}{l}\text { Understanding students' sports level from the side, A2.2 } \\
\text { Observing students' movement in and out of class, A2.3 }\end{array}$ \\
\hline & & Suitable for students' affordability, A3.1 \\
\hline & Exercise load analysis, A3 & $\begin{array}{c}\text { Analyzing students' physical performance in real time, A3.2 } \\
\text { The exercise load arrangement has consistency, A3.3 }\end{array}$ \\
\hline & $\begin{array}{c}\text { Selection of teaching content, } \\
\text { A4 }\end{array}$ & $\begin{array}{l}\text { The teaching content is targeted, A4.1 } \\
\text { There is continuity in what is being taught, A4.2 } \\
\text { In line with the emotional experience of students, A4.3 }\end{array}$ \\
\hline \multirow{5}{*}{ The practice teaching, $\mathrm{B}$} & $\begin{array}{l}\text { Theoretical knowledge of } \\
\text { motion, B1 }\end{array}$ & $\begin{array}{l}\text { Mastering the exercise methods learned in class, B1.1 } \\
\text { Mastering the essentials of sports skills learned in class, B1.2 }\end{array}$ \\
\hline & Sports skills, B2 & $\begin{array}{l}\text { Enhancing individual sports skills, B2.1 } \\
\text { Cooperative sports skills improved, B2.2 }\end{array}$ \\
\hline & Physical, B3 & $\begin{array}{l}\text { Body shape improvement, B3.1 } \\
\text { Physical improvement, B3.2 }\end{array}$ \\
\hline & $\begin{array}{l}\text { Experience of body and mind, } \\
\text { B4 }\end{array}$ & $\begin{array}{c}\text { Participating in the process of sports, having a happy psychological } \\
\text { experience, B4.1 } \\
\text { Feeling physical improvement, B4.2 }\end{array}$ \\
\hline & Sports participation, B5 & $\begin{array}{c}\text { Consciously participating in physical activities in and out of class, B5.1 } \\
\text { High enthusiasm during exercise, B5.2 } \\
\text { Taking initiative to apply your knowledge and skills, B5.3 }\end{array}$ \\
\hline
\end{tabular}

the judgment matrix obtained by multiple experts will be generated. Finally, according to the judgment matrix of each expert, the group judgment matrix will be synthesized.

If a judgment matrix has the following properties, the judgment matrix has complete consistency: (a) $b_{i j}=1$; (b) $b_{i j}=(1 / b) ;(\mathrm{c}) b_{i j}=\left(b_{i k} / b_{k j}\right)(i, j, k=1,2, \ldots, n)$.

Therefore, as long as $b_{11}$ and $b_{12}, \ldots, b_{1 n}$ are given by experts, a complete judgment matrix can be constructed according to their consistency. However, the judgment matrix generated in this way is not necessarily the expert's opinion, and the expert may have other results, so the expert can continue to modify the weight generated by the system, knowing that it is in line with the expert's own opinion. This not only reflects the opinions of experts, but also makes the final judgment matrix pass the consistency test as far as possible.

(2) The Calculation Process of Hierarchical Single Sort. The judgment matrix only gives the degree of relative importance of an index at the upper level to some indexes at the next level. According to the analytic hierarchy process (AHP), it is necessary to rank each judgment matrix at a single level and then calculate the weight of each judgment matrix. The process of AHP is explained via Figure 1. The calculation of hierarchical single sort is actually to calculate the eigenvalues and eigenvectors of the judgment matrix.

The judgment matrix is set as $C$, and the eigenvalue and eigenvector of matrix $C$ are calculated; that is, we calculate the formula $\mathbf{C W}=\boldsymbol{\lambda}_{\max } \mathbf{W}$, in which $\boldsymbol{\lambda}_{\max }$ is the maximum feature root of matrix $C$, and $W$ is the corresponding eigenvector.

At present, there are mainly three methods to calculate the maximum eigenvalue and eigenvector of judgment matrix: sum product method, square root method, and power method. The specific steps are as follows:

(a) Normalization by column: normalize each column of the judgment matrix $C$; that is, $\overline{b_{i j}}=\left(b_{i j} / \sum_{k=1}^{n} b_{i j}\right),(i, j, \ldots, n)$.

(b) Sum by row: the matrix is obtained by addition and normalization by row; i.e., $\overline{\omega_{i}}=\sum_{j=1}^{n} \overline{b_{i j}}(i, j, \ldots, n)$, and $\bar{W}=\left(\overline{\omega_{1}}, \overline{\omega_{2}}, \ldots, \overline{\omega_{n}}\right)$.

(c) Calculate $W$ : normalize $\overline{\omega_{i}}$ obtained in the previous step; that is, $W_{i}=\left(\overline{\omega_{i}} / \sum_{j=1}^{n} \overline{\omega_{i}}\right), i, j=1,2, \ldots, n$; then, the eigenvector can be obtained as $W=\left(W_{1}, W_{2}, \ldots, W_{n}\right)^{T}$.

(d) Calculate the maximum eigenvalue of the judgment matrix $C: \lambda_{\max }=(1 / n) \sum_{n}^{1}(B W)_{i} / W_{i}$.

(e) Verify the consistency of judgment matrix $C$.

Definition 1. If $\lambda_{\max }=n, b_{i k} \times b_{k j}=b_{i j}$, then the matrix $C$ is consistent. In order to test the consistency of the judgment matrix $C$, we need to calculate its consistency index, which is used to test the consistency of experts' judgment thinking of setting weights. The calculation formula is as follows: $\mathrm{CI}=\left(\left(\lambda_{\max }-n\right) /(n-1)\right)$.

Obviously, when $\mathrm{CI}=0$, the judgment matrix $C$ has complete consistency. With the increase of CI value, its consistency will be worse and worse; that is, the value of $\mathrm{CI}$ is inversely proportional to the consistency. In order to test whether the matrix $C$ has qualified consistency, we need to get the average ratio index (RI) of the judgment matrix and then compare it with CI. 


\begin{tabular}{|c|c|}
\hline STEP 1 & Identify Objectives \\
\hline \hline STEP 2 & Identify Criteria, Sub-Criteria And Intensity Level \\
\hline \hline STEP 3 & Identify Alternatives \\
\hline \hline STEP 4 & Draw Hierarchal Tree \\
\hline \hline STEP 5 & Make Pair-wise Comparsion Between Criteria, Sub-Criteria Based \\
\hline \hline STEP 6 & On Their Intensity Level \\
\hline \hline STEP 9 & Make Pair-wise Comparsion Between Alternatives Based On Ratio \\
\hline \hline STEP 7 & Scale \\
\hline
\end{tabular}

FIgURE 1: Process of AHP.

Finally, the consistency ratio of the judgment matrix $C$ is calculated by the formula $\mathrm{CR}=(\mathrm{CI} / \mathrm{RI})$, where $\mathrm{RI}$ is determined from Table 2 .

When $\mathrm{CR}<0.1$, it is considered that the judgment matrix has qualified consistency; that is, the index weight obtained by experts' comparison is reasonable, and the conclusion obtained by the analytic hierarchy process is scientific. Otherwise, it is necessary to reconstruct the judgment matrix until the qualified consistency requirement is obtained.

Other obtained judgment matrices should also be calculated according to this method until all of them are processed. Finally, the weights of the index layer relative to the criterion layer and the criterion layer corresponding to the target layer are obtained. The results are shown in Table 3.

\subsection{Analysis of Teaching Evaluation Index System of Public Physical Education}

\subsubsection{Teachers' Teaching Preparation}

(1) Site Facilities Inspection. Sports venues and related teaching facilities are the premise and material conditions for physical education and students' extracurricular exercise and are important factors to ensure the smooth development of physical education in colleges and universities. In 1990, the State Education Commission promulgated the "Regulations on School Physical Education Work," and in 2002 the Ministry of Education issued the "Guidelines for National College Physical Education Curriculum," which pointed out that schools at all levels should gradually be equipped with the necessary venues, equipment, and facilities for physical education and included in the teaching equipment supply plan. It can be seen that the Ministry of Education and National Government Departments attach great importance to physical education teaching but also attach great importance to safety work. In the physical education teaching work in colleges and universities, we should pay attention to the layout of venues and equipment and always check the safety of facilities [10], so as to make the layout of teaching facilities scientific and reasonable. The location and distance of equipment and teaching facilities should be scientific and reasonable. At the same time, it should be considered that not only the needs of students' comprehensive physical exercise should be met, but also the equipment should be adjusted very conveniently, so as to eliminate potential safety hazards and avoid safety accidents. This is not only the need of physical education in colleges and universities, but also the need of being people-oriented and the need of modern sports reform and development. 
TABLE 2: Saaty's derived ratio index (RI) against value of $n$.

\begin{tabular}{|c|c|c|c|c|c|c|c|c|c|c|c|c|c|c|}
\hline 1 & 2 & 3 & 4 & 5 & 6 & 7 & 8 & 9 & 10 & 11 & 12 & 13 & 14 & 15 \\
\hline 0.00 & 0.00 & 0.58 & 0.90 & 1.12 & 1.24 & 1.32 & 1.41 & 1.45 & 1.49 & 1.51 & 1.48 & 1.56 & 1.57 & 1.59 \\
\hline
\end{tabular}

Table 3: Determination of weights of teaching evaluation index system.

\begin{tabular}{|c|c|c|c|c|}
\hline Level 1 indexes & Level 2 indexes & Level 3 indexes & $\begin{array}{l}\text { Index weight } \\
\text { score }\end{array}$ & Weight sorting \\
\hline \multirow{11}{*}{$\begin{array}{l}\text { Teacher's preparation for } \\
\text { teaching, A }\end{array}$} & \multirow[b]{2}{*}{$\begin{array}{l}\text { Inspection of site } \\
\text { facilities, A1 }\end{array}$} & Site safety inspection, A1.1 & 0.89 & 1 \\
\hline & & $\begin{array}{c}\text { The number of teaching facilities required is } \\
\text { adequate, A1.2 }\end{array}$ & 0.78 & 2 \\
\hline & \multirow{3}{*}{$\begin{array}{l}\text { Mastery of exercise level, } \\
\text { A2 }\end{array}$} & Talking to students in and out of class, A2.1 & 0.88 & 1 \\
\hline & & $\begin{array}{l}\text { Understanding students' sports level from the side, } \\
\qquad \text { A2.2 }\end{array}$ & 0.76 & 3 \\
\hline & & $\begin{array}{l}\text { Observing students' movement in and out of class, } \\
\text { A2.3 }\end{array}$ & 0.82 & 2 \\
\hline & \multirow{3}{*}{ Exercise load analysis, A3 } & Suitable for students' affordability, A3.1 & 0.86 & 1 \\
\hline & & $\begin{array}{c}\text { Analyzing students' physical performance in real } \\
\text { time, A3.2 }\end{array}$ & 0.83 & 2 \\
\hline & & $\begin{array}{l}\text { The exercise load arrangement has consistency, } \\
\text { A3.3 }\end{array}$ & 0.81 & 3 \\
\hline & \multirow{3}{*}{$\begin{array}{l}\text { Selection of teaching } \\
\text { content, A4 }\end{array}$} & The teaching content is targeted, A4.1 & 0.88 & 1 \\
\hline & & There is continuity in what is being taught, $\mathrm{A} 4.2$ & 0.76 & 3 \\
\hline & & $\begin{array}{l}\text { In line with the emotional experience of students, } \\
\text { A } 4.3\end{array}$ & 0.85 & 2 \\
\hline \multirow{11}{*}{ The practice teaching, $\mathrm{B}$} & \multirow{2}{*}{$\begin{array}{l}\text { Theoretical knowledge of } \\
\text { motion, B1 }\end{array}$} & $\begin{array}{l}\text { Mastering the exercise methods learned in class, } \\
\text { B1.1 }\end{array}$ & 0.85 & 1 \\
\hline & & $\begin{array}{l}\text { Mastering the essentials of sports skills learned in } \\
\text { class, B1.2 }\end{array}$ & 0.81 & 2 \\
\hline & \multirow{2}{*}{ Sports skills, B2 } & Enhancing individual sports skills, B2.1 & 0.87 & 1 \\
\hline & & Cooperative sports skills improved, B2.2 & 0.84 & 2 \\
\hline & \multirow{2}{*}{ Physical, B3 } & Body shape improvement, B3.1 & 0.82 & 2 \\
\hline & & Physical improvement, B3.2 & 0.85 & 1 \\
\hline & \multirow{2}{*}{$\begin{array}{l}\text { Experience of body and } \\
\text { mind, B4 }\end{array}$} & $\begin{array}{l}\text { Participating in the process of sports, having a } \\
\text { happy psychological experience, B4.1 }\end{array}$ & 0.89 & 1 \\
\hline & & Feeling physical improvement, $\mathrm{B} 4.2$ & 0.83 & 2 \\
\hline & \multirow{3}{*}{ Sports participation, B5 } & $\begin{array}{c}\text { Consciously participating in physical activities in } \\
\text { and out of class, B5.1 }\end{array}$ & 0.85 & 1 \\
\hline & & High enthusiasm during exercise, B5.2 & 0.81 & 2 \\
\hline & & $\begin{array}{c}\text { Taking initiative to apply your knowledge and } \\
\text { skills, B5.3 }\end{array}$ & 0.76 & 3 \\
\hline
\end{tabular}

Therefore, in college physical education teaching, teachers and other relevant staff checking the safety of teaching facilities before and during class, as well as the reasonable layout of equipment, can effectively protect the physical safety of students [11], so that students' sports skills and theoretical knowledge learning have no more worries, so as to further reflect the learning effect, improve students' learning effect, and then improve the performance of physical education teaching. It can be seen from the questionnaire that the safety inspection score of the venue is the highest, with a score of 0.89 . Therefore, we can see the importance of the safety inspection of the venue, and we should pay attention to the evaluation results when we evaluate the performance of physical education. In addition, the score of the index of sufficient number of teaching facilities is 0.78 , which is the lowest under this secondary index. It can be seen that whether the number of teaching facilities is sufficient or not is the relative interference factor affecting the improvement of physical education teaching performance.

(2) Mastery of Sports Level. The so-called sports level refers to people's own skill level when they participate in sports and training. It is the comprehensive performance of people's physical quality and various organs in sports. The level of exercise not only depends on the energy supplied by metabolism, but also depends on the perseverance and endurance of people. Physical education teachers' understanding and mastery of students' sports level are particularly important for physical education teaching in colleges and universities. Through the data statistics, we can see that the score of talking with students in and out of class is 0.88 , which is the first third-level index in the second-level index. Therefore, through the way of talking with students in and out of class, physical education teachers can better understand the actual sports level of students, so as to develop 
more targeted teaching content and teaching plan and improve the performance of physical education teaching from a student-centered perspective. We can see that experts attach great importance to observing students' sports in and out of class. Because students' lack of sports theory knowledge and sports experience may affect students' judgment of their own sports level, teachers can more directly understand students' actual sports ability and improve teaching performance by observing students' sports situation in and out of class.

(3) Analysis of Exercise Load. Exercise load, also known as physiological load, is the load that people have to bear when they exercise. It includes the amount and intensity of exercise. The main influencing factors of exercise intensity are speed and load. For example, college students' heart rate can reach about 130 beats/min immediately after 100 meter full speed running, and the heart rate is generally about 100 beats/min after 400 meter jogging. From this example, we can see that the intensity of 100 meter fast running is relatively high, while that of 400 meter jogging is relatively low. The amount of exercise refers to the energy consumed by people in sports, which is determined by the duration and characteristics of sports [12]. Exercise time refers to the total time or the interval of each exercise in the process of physical education. The duration or interval of exercise directly determines the size of exercise load. In the teaching of physical education, if the students take a long time of highintensity exercise, the load of the body will appear too large.

The difficulty of teachers' arrangement of physical education teaching content, as well as the appropriateness of teaching organization form and method, will affect the size of sports load. For example, the lack of sports venues and equipment in physical education teaching will lead to the long interval of students' practice, resulting in too small exercise load, which cannot achieve the purpose of physical exercise. However, if the duration of practice is too long and the interval is too short, this will easily cause the students to bear too much exercise load, which is beyond the limit of the students' physical and mental health at that age stage and has a negative effect on the students' physical and mental health and the completion of teaching cooperation goals. Therefore, in the process of physical education teaching, we should learn to reasonably arrange and regulate the exercise load. A more suitable exercise load can have a better exercise effect. However, with the improvement of students' comprehensive quality, according to the students' ability to bear the exercise load, we can also gradually and rhythmically increase the load [13].

The physical education teachers in colleges and universities should arrange the exercise load according to the students' physical ability before class and pay attention to the scientific arrangement of textbooks with different exercise nature, varying intensity and suitable density. In the selection of teaching material content, we can arrange large load and small load exercise alternately.

The sports load of physical education includes standard model, "low in former and high in later" model, and "high in former and low in later" model. The standard model refers to the arrangement of sports practice load gradually so that it increases from small to a certain level, lasts for a period of time, and then gradually decreases. The "low in former and high in later" model refers to the gradual increase of sports practice load from small to large. For example, the former part of a physical education class is a new curriculum, and the latter part is a review curriculum [14]. The mode of "high in former and low in later" means that, in the basic part of physical education, the practice load of the former part is larger and the practice load of the latter part is smaller. In the process of physical education, the regulation of sports load should be a combination of dynamic and static exercise, alternating high and low intensity of sports load, with medium intensity being the main one, and at the same time taking into account the students' recovery after class.

In the process of physical education teaching, the relatively simple load measurement methods are pulse measurement method and observation method. When measuring the pulse, the teacher can judge the exercise load according to the pulse of the students. In general, college students' heart rate should be 120-140 beats/min to achieve the best effect. When using the observation method, physical education teachers can judge the exercise load by observing the students' expression, panting, sweating, and so on. The observation method can directly and easily detect the students' exercise load. For example, when students bear a small load of exercise, their body reaction will be slight sweating, and their face will turn red slightly; when they bear a medium intensity load, their face will turn red, and there will be sweat drops on their body surface; when they bear too much intensity load, their face will turn white, and their action will be slow. Therefore, the arrangement of exercise load should be based on students' physical endurance and attention to students' physical and mental experience.

From the questionnaire, we can see that the score of teaching content design suitable for students' affordability is 0.86 . Under this secondary index, the score is the highest. Therefore, we can draw a conclusion that experts attach great importance to the index stating that the design of teaching content is suitable for students' affordability. Therefore, in the evaluation of physical teaching performance in colleges and universities, we should consider whether the design of teaching content is suitable for students' affordability as a key consideration and trade-off index; and for the feedback of the assessment results, we should also carefully analyze and judge whether the assessment results are excellent or not, and we should constantly improve work performance. The score of real-time attention to students' physical performance is 0.83 , and it is ranked second. Therefore, whether teachers can always pay attention to students' physical performance and reaction in the teaching process can also be the focus of performance appraisal. The score of consistency of exercise load arrangement is 0.81 , but it can be seen from the score that the periodical arrangement of exercise load is not highly recognized by experts, which has something to do with the difficulty of quantifying the periodical exercise load and the actual operation. Therefore, in the class plan, the periodic 
arrangement of exercise load may not be very obvious for improving the classroom teaching performance of colleges and universities. However, in the medium and long term, there is still certain enthusiasm for arranging the exercise load in stages.

(4) Teaching Content Screening. The content of physical education teaching is the data information transmitted in the process of teaching. Under the background of the new curriculum reform, physical education teaching content is also considered as the material and information of interaction between teachers and students in the teaching process in order to achieve the teaching purpose. Physical education teachers' choice of physical education classroom teaching content will largely determine the enthusiasm of students for classroom learning [15] and also affect the performance of physical education teaching in colleges and universities to a certain extent. Therefore, the reasonable selection of physical education teaching content is very important. From the data shown in the questionnaire, we can see that the teaching content has continuity and the score is the lowest under this secondary index. Therefore, in the process of teaching performance evaluation, physical education teachers should put more energy into the direction of classroom teaching and pay more attention to the cultivation of students' comprehensive physical quality and sports ability. The score of students' emotional experience is 0.85 . It can be learned that the experts are very concerned about the teaching content in line with the students' interest in sports, so when there is a choice, they should choose as much as possible the content that the students are interested in, which will help to improve the students' learning effect. The teaching content with pertinence has the highest score. It also reflects the importance of the pertinence of the teaching content to the teaching performance. Therefore, we should pay more attention to the pertinence of the teaching content when we evaluate the performance of physical education teaching in colleges and universities and take this as an important evaluation index.

\subsubsection{Practical Teaching}

(1) Sports Theory Knowledge. Different from sports skills, motor theoretical knowledge is instructive and abstract content in the process of physical exercise. For example, after exercise, the body will experience fatigue, which is manifested as muscle strength decline, muscle stiffness, swelling and pain, and slow and uncoordinated movement. After the emergence of fatigue, we can promote the elimination of fatigue by supplementing the substances consumed by the body in sports, such as supplementing nutrients and using some traditional Chinese medicine to regulate the body function. This way of promoting fatigue recovery belongs to the category of sports theory knowledge. Through the questionnaire data, we can see that the score of mastering the sports technology essentials learned in class is 0.81 , ranking first in the same three-level index. This shows that the main sports skills of physical education are obtained under the guidance and help of physical education teachers; that is to say, the knowledge learned in class accounts for a considerable proportion of what students learn. The mastery of extracurricular sports technology is more completed by students themselves [16]. To master the exercise methods learned in class, students can use the scientific exercise methods learned to strengthen physical exercise and enhance physical quality when they participate in extracurricular or social exercise.

(2) Sports Skills. Sports skill refers to the body's ability to complete body movements acquired in sports, including the cooperation ability between different muscle groups, that is, the ability to mobilize muscle groups to work. Its formation has to go through several specific stages of muscle slow response, differentiation, stability, and automation. These stages gradually progress in the formation process of motor conditioned reflex. The formation and development of sports skills are affected by many factors, including training methods, training basis, training purpose, and training enthusiasm. The formation of sports skills is phased, and each stage has different characteristics. Generally speaking, the formation of sports skills can be divided into three stages. One is the cognitive stage of action. At the beginning of skill learning, the neural process of the practitioner is in the stage of categorization, the scope of attention is narrow, the accuracy of perception is low, there is uncoordinated connection between actions, especially the degree of muscle relaxation, the whole action appears to be busy and tense, and the completed action is not accurate [17]. At this stage, practitioners primarily perform imitation through visual observation, with vision being used to regulate the action more frequently. As a result, the action is not well controlled; it is difficult to identify their own flaws and errors. The second is the contact stage of action. After a period of action practice, the exerciser initially masters some actions and begins to connect these actions consciously. At this time, the neural process of the exerciser begins to convert into differentiation inhibition; that is to say, only under the condition of stimulation can the conditioned response be induced, while the approximate stimulation does not cause the conditioned response. In the contact stage of action, the process of excitation and inhibition is more accurate in space and time, and the scope of attention is relatively wider; the degree of tension will also be reduced, the interaction between actions will be gradually eliminated, the accuracy of actions will be improved to a certain extent, and some action skills will be initially formed. In the stage of action connection, the practitioner can judge by logical thinking, summarize the essential characteristics of action, and connect some scattered actions into a whole action. The third is the perfect stage of action. At this stage, the movement of the practitioner will establish a stable dynamic pattern in the brain, and the excitation and inhibition of the neural process will be more accurate. Each movement can be displayed in a complete series, and the degree of automation will be further expanded. At this stage, the practitioners' attention scope is expanded, which is mainly used for the processing of environmental information; the degree of attention to the 
action itself is reduced; and the wrong action can be found and corrected in time [18].

In the process of physical education teaching in colleges and universities, we should also pay attention to the stage characteristics of the mastery of sports skills and carry out physical education according to the characteristics of each stage to improve teaching performance. There are two aspects in the improvement of sports skills: one is the improvement of personal motor ability, and the other is the improvement of cooperation skills with others. The former pays more attention to personal factors, while the latter pays more attention to students' physical exercise ability. The data shows that the improvement of individual sports skills is 0.87 , higher than that of cooperative sports skills, which shows that, regarding the issue of sports skills, experts are relatively more inclined to improve individual sports skills. This also indicates that the improvement of individual sports skills is the basis and premise of cooperative sports skills.

(3) Body Constitution. Through the data statistics, we can see that the body shape improvement score is 0.82 and the physical fitness enhancement score is 0.85 . We can also see that the main aspect of physical enhancement is not the improvement of external body shape, but the improvement of physical fitness and physical function level. Therefore, in the performance evaluation of physical education teaching, we should pay more attention to physical fitness indexes.

(4) Physical and Mental Experience. Physical and mental experiences refer to the individual's self-emotional experience of his own attitude and emotion based on his own value judgment and evaluation. Physical and mental experiences constitute an advantage of physical education in colleges and universities compared with other subjects. In the process of physical education learning, students can feel both physical and psychological feelings. In the process of participating in sports, the score of psychological happy experience is 0.89 , and the score of physical improvement is 0.83 . Comparing the two, in sports evaluation, we should pay more attention to students' psychological experience and feeling, so that students can maintain a good state of mind when they exercise.

(5) Sports Participation. Sports participation refers to students' enthusiasm and interest in participating in sports learning in and out of class. If students are not interested in sports activities, they will not actively participate in sports activities. Therefore, the cultivation and stimulation of students' interest in sports should be put in the first place in college physical education. Whether it is the choice of teaching content or teaching methods, we should pay attention to the stimulation of students' enthusiasm for sports participation. In order to cultivate and stimulate students' interest and hobby in participating in sports activities, it is necessary to emphasize that students should be the main body in sports teaching, let students gain autonomous learning and cooperative learning, and let students experience the process of achieving results both physically and mentally, so as to improve students' interest in sports and enthusiasm for participation [19]. Teachers should put the development of students at the center of every link, such as the establishment of teaching objectives, the selection of teaching contents, and the application of teaching methods, so as to fully tap their learning potential and promote the allround development of students. Through the survey data, we can see that the weight score of consciously participating in sports activities in and out of class is 0.85 , the weight score of high enthusiasm in the process of sports is 0.81 , and the weight score of actively applying the learned knowledge and skills is 0.76 . Consciously participating in physical activities is an important index for the evaluation of physical education, because it can not only promote the development of physical education in colleges and universities, but also promote students' development of the habit of lifelong exercise. The enthusiasm about participation and the active application of the knowledge and skills learned are also very important for the evaluation of physical education work. We can understand the role of physical education teachers in the classroom from the side and provide some references for the improvement of teachers' teaching.

2.4. Target Evaluation. Target evaluation is the last link of target management in the teaching process of colleges and universities, and it is the main means to test the effect of target management in all teaching colleges and departments. Target evaluation must be objective and fair, and its center is to establish a scientific and reasonable evaluation mechanism and use unified and standardized evaluation methods. In the evaluation of each teaching department, it is necessary to formulate evaluation methods strictly in accordance with the "evaluation index system of teaching process objectives" of that year, determine evaluation points for specific objectives, set different scores according to different degrees of reaching the objectives, clarify the source channel of data, and have corresponding basic data as the support of scoring standards, so as to achieve the goal of quality. For qualitative indexes, we should also make the way of evaluation clear and strive to make the target evaluation reasonable and standardized. In terms of evaluation methods, there are three parallel links: self-examination and self-evaluation, evaluation of main functional departments, and evaluation of school evaluation team. As far as the teaching management is concerned, the department in charge of teaching in colleges and universities (academic affairs office) has a better understanding of the teaching work of each department, and the information they have mastered is more detailed and accurate. Therefore, the academic affairs office should be responsible for evaluating and summarizing the completion of its relevant work objectives, and the results will be relatively objective and accurate.

\section{Teaching Reform Strategy of Public Physical Education}

3.1. Comprehensive Reform Objectives. Based on the outline, we should strive to achieve the goal of public physical education curriculum through reform, and focus on the 
achievement of skill goal, so as to promote the effective achievement of other curriculum goals.

3.2. Comprehensive Reform Ideas. The level of students' sports skills is the core problem that affects the educational goal of public physical education curriculum. To establish a correct and clear concept of teaching sports skills as the core can solve the outstanding contradiction between multiple objectives and less teaching hours of public physical education curriculum as the focus and achieve the goal of skills as a breakthrough and focus. Through improving students' physical exercise skills, students can get the fun of participation and experience the feeling of success in physical exercise [20], stimulate their initiative and enthusiasm for consciously participating in sports, develop the habit of conscious exercise, and gradually form good behavior habits and healthy way of life.

\subsubsection{Multiobjective Realization of Public Physical Education.} Can the public sports curriculum achieve the goal of the outline? Theory and practice have proved that only relying on physical education cannot fully achieve these goals. For example, for the goal of physical health, according to the law of excessive recovery of sports human science, once-a-week physical education, even if it is completely turned into a physical fitness training curriculum, cannot effectively improve students' physical health; the development of students' mental health and social adaptability must be based on students' effective participation in sports activities. If students do not have the ability to effectively participate in sports activities, it is obviously difficult to achieve only relying on public sports courses.

3.2.2. Teaching Focus of Public Physical Education. What should physical education do? To answer this question, we must find the logical connection between goals. The logical starting point is sports participation, because participation is the premise, and strengthening students' physique, improving physical and mental health, and improving social adaptability are the results of participation. Sports skills are the means of sports participation and the bridge to the above goal. From this logical chain, we can see that, to achieve the important result goal, the core problem is not the result goal itself, but the two conditional goals of sports participation and skills. Conditions and objectives are the key points.

There is an internal causal relationship between sports participation and sports skills. The motivation of sports participation is gradually formed in the learning and application of sports skills. The greater the progress of sports skills is, the higher the level is, the more the positive energy is, and the more stable the interest of sports participation is. In turn, strong interest promotes the improvement of sports skills with greater motivation. This has also been well confirmed in reality. In the sports field, those students who are willing to exercise are basically students with a certain level of skills. Thus, sports skill is the main aspect of the contradiction in the core problem.
Our understanding of physical education has changed from "physical development education" to sports based education. Skill is the basis for the effective realization of sports. Therefore, the teaching focus of public physical education is to help students achieve the conditional goal of imparting sports skills. This is also in line with the characteristics of physical education. Among the five goals, from the perspective of teaching and learning, sports skills are the most difficult and the most needed. This paper designs a comprehensive reform plan of public physical education curriculum teaching, which is shown in Figure 2.

\subsection{Key to Comprehensive Reform.}

(1) In order to ensure the quality and level of "outline" curriculum objectives, management by objectives is used to replace the stage evaluation by semester. "Management by objectives" is to set up the items that must be passed in college physical education according to the spirit of "outline" and credit regulations. All items will no longer be repeated in stages according to the semester. Students only need to participate in the level examination in the sense of ultimate goal, and each passing item will get 1 academic point. The comprehensive evaluation standard is formulated according to the achievement level required by the outline. The prominent feature of "management by objectives" is that it breaks the regulations of semester and academic year. Students can take the proficiency test within four years of university. If they fail to pass the test, there is no need for make-up or retake test. They just need to take the proficiency test again until they pass it.

The implementation of "management by objectives" has solved two problems: One is the problem of time. For most students, two years and four semesters are not enough time to reach the level required by the outline. The second is the problem of evaluation standard. It is very difficult to reasonably divide it into four semesters according to the achievement level required by the outline and ensure the final completion. The results of longterm practical operation show that the effect of periodic evaluation with semester as cycle is generally " $1+1<2$ "; that is, the sum of segmented learning effect of each semester cannot reach the level that the cumulative learning effect should reach, so the final achievement level is not satisfactory.

(2) To improve teaching efficiency and promote students' active learning, we should focus on students and make an appointment for learning on demand. "Booking learning on demand" means that students decide the learning time of physical education according to the requirements of management by objectives and their own level. They can make an appointment according to the semester as the time 


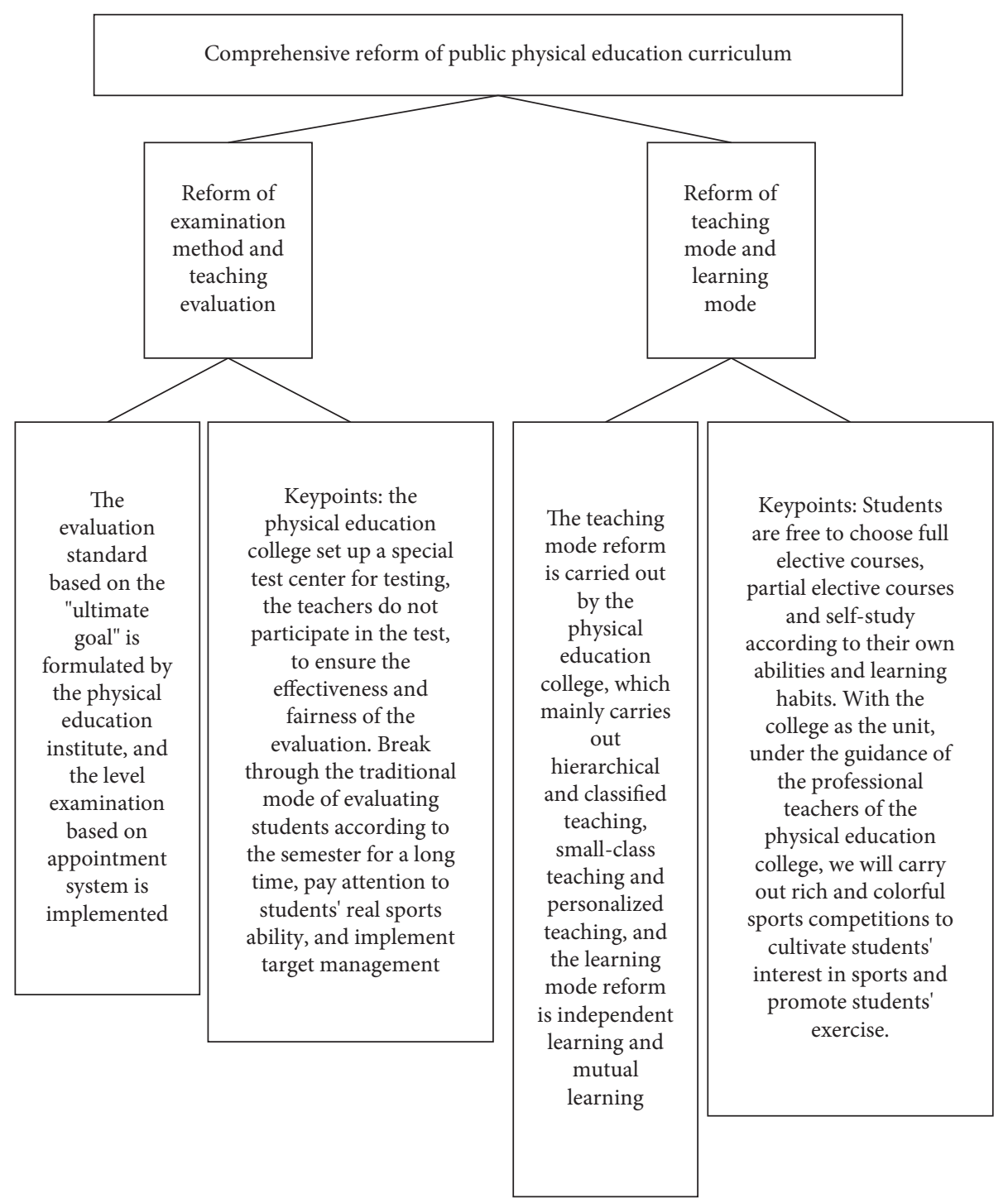

Figure 2: Comprehensive reform plan of public physical education curriculum.

unit. They can study for less or more than 4 semesters. Students can make an appointment for 6 semesters free of charge. After 6 class hours, they can continue to make an appointment according to the credit payment.

"Booking learning on demand" truly reflects the belief that students are the main body, giving students the initiative of learning and providing sufficient curriculum platform for students' learning at the same time. This flexible mechanism brings two advantages. One is to ease the contradiction that teaching resources cannot meet the needs of options. If students cannot choose the project they like in this semester, they do not have to choose the curriculum. They can choose the curriculum again in the next semester. In this way, they can choose to study according to their interests to the maximum extent. The second is that it is conducive to the cultivation of students' habit of active learning. Appointment learning reflects the students' independent choice and gives the students the right of learning autonomy. The students in class come with needs, and they have high enthusiasm and initiative in class, which is conducive to improving the teaching effect.

(3) The term examination is changed into an appointment examination to build a platform for the realization of target management. "Appointment examination" means that students decide when to take the level examination according to their own learning and training level.

(4) The implementation of the separation of teaching and examination makes the examination more fair, just, and accurate and ensures the quality of the examination. The college of physical education can set up a public physical education curriculum examination center. After the students make an 
appointment, the examination center will uniformly organize the appointment examination, so as to ensure the fairness and quality of the examination. Due to the long opening period of the appointment examination, the number of references can be effectively divided, and the order of the separation of teaching and examination is guaranteed.

(5) In terms of teaching content, we should enrich the teaching content of public physical education curriculum in colleges and universities, so that it can not only meet the unique physiological and psychological characteristics of college students, but also meet the needs of life and work of high-quality talents after graduation. Teaching content is the most direct embodiment of the goal of physical education in colleges and universities, and it is also the concretization of the goal of physical education in colleges and universities, serving for the realization of the goal of physical education. Therefore, the teaching content of public physical education in colleges and universities should be personalized and diversified. In the teaching process, students should be put in the core position, and students' interest in sports and habit of lifelong physical exercise should be cultivated, so that students can master one or more sports skills that can be practised for a long time. The teaching content should highlight the functionality, fitness, entertainment, practicality, and lifelong development and strive to make students adapt to the requirements of social sports for their knowledge and skills after graduation, so that physical exercise has gradually become an indispensable habit in college students' life and physical fitness activities have gradually become one of their lifelong pursuits.

(6) In the selection of teaching materials, we should pay attention to the content of teaching materials, closely adhere to the teaching objectives, and choose the corresponding teaching materials according to the characteristics of college students and the needs after graduation. At the same time, the content of teaching materials should also pay attention to interest. When introducing learning methods, practice methods, competition methods, etc., they should be accompanied by rich illustrations, so as to attract college students to consciously read and consciously refer to the teaching materials for exercise, so as to make the teaching materials "good teachers and helpful friends" for college students to exercise. Finally, the content of teaching materials should be in line with lifelong sports, focusing on introducing lifelong sports ideas, strengthening students' fitness awareness, adding practical content, including the rules of international competitions, presenting relevant knowledge of strengthening physique, maintaining body and mind, demonstrating sports injury treatment knowledge, and so on. In a word, the teaching materials of general public physical education should start from the perspective of education; closely adhere to the teaching objectives; fully reflect the significance of the dominant teaching materials of sports culture-the function of fitness and sports skills training and the significance of hidden teaching materials-and the role of lifelong sports consciousness, as well as the socialized training and emotion of students; establish a teaching system that students are interested in and conform to the actual life of students and be conducive to guiding students after graduation lifelong physical exercise.

(7) The concept of teachers should also be changed. Teachers of public physical education curriculum in colleges and universities are the backbone of organizing and implementing physical education teaching, scientific research, and teaching reform. Their ideas play an important role in the smooth transformation of college physical education from stage to lifelong physical education. The concept of college physical education teachers cannot be formed overnight. On the one hand, it needs regular training of modern physical education thoughts and theories for physical education teachers in colleges and universities, so that they can take the lead in establishing lifelong physical education consciousness on the basis of full learning and understanding of lifelong physical education thoughts and theories, so as to promote the transformation of their ideas. On the other hand, with the rapid development of scientific and technological knowledge and the increasingly perfect knowledge system of sports science today, physical education teachers cannot be satisfied with the status quo, being immersed in the past understanding of college physical education. They should be depend on the present but also face the future; timely learn new sports knowledge; timely master new sports skills; grasp the trend of college physical education development, in order to meet the needs of students learning and entertainment needs; and constantly broaden the horizons of students, so that physical education becomes more popular among students. At the same time, they should spread ideas to students and effectively improve the quality of life of students.

At the same time, the quality of college physical education teachers should be improved. Public physical education teachers in colleges and universities must have profound theoretical knowledge, good moral quality, solid professional skills, and innovation ability. In order to adapt to the rapid development of modern education, college public physical education teachers should timely update and expand their existing professional knowledge; actively learn the knowledge of Internet of Things engineering technology, life science, medicine, biology, physics, and other related disciplines; 
improve their knowledge structure and the quality of science and culture; and flexibly use all kinds of knowledge in the teaching process, so as to meet the needs of students. This can satisfy the students' thirst for knowledge and stimulate their interest in learning sports. At the same time, college teachers should master modern physical education, scientific research means, and technology; have a certain innovation ability to teach and do scientific research; use a variety of forms of teaching; spread the latest sports information to students; and fully mobilize the enthusiasm of students for learning sports, so that students will not have resistance to public sports curriculums because of monotony, which is more conducive to students' health. The formation of the consciousness of physical education and the habit of physical exercise can further promote the all-round development of students' body and mind.

Finally, a new type of teacher-student relationship should be established. Under the guidance of lifelong physical education, teachers of public physical education in colleges and universities should establish equal, mutual-trust, intimate, interactive, stable, and lasting teacher-student relationship with students. In the process of organization, management, and implementation of public physical education curriculum, physical education teachers should always remember to restrict and regulate their words and deeds according to teachers' professional ethics standards; pay attention to communication and interaction with students; and teach students in accordance with their aptitude, so as to meet the learning needs of different students, no longer being a "sage" on the platform, but a good teacher and friend of students, and finally to teach students by their own words and deeds.

(8) The propaganda and education of lifelong sports consciousness should be strengthened. To cultivate and strengthen college students' lifelong sports awareness, it is necessary to strengthen the publicity and education of lifelong sports awareness. Colleges and universities can provide propaganda and education of sports knowledge to college students through various channels such as billboards, radio stations, and school websites, so as to improve their cognition of sports, expand their horizons of sports knowledge, stimulate their enthusiasm for sports, and encourage them to actively participate in physical exercise. Colleges and universities can also invite students, their parents, and people from all walks of life to participate by holding various sports knowledge lectures on a regular basis, so as to expand the social influence of college sports. Publicity and education can help college students to establish the concept of "lifelong sports" and make them understand that sport is an organic part of their study, life, and work and is the support and guarantee of their lifelong health and happy life, so as to develop good physical exercise habits, form a healthy and happy lifestyle, and really do one hour of physical exercise every day.

(9) Attention should be paid to the formation of students' awareness of lifelong physical exercise. Adhering to the guiding ideology of "people-oriented, health first," the public physical education curriculum should establish a three-dimensional health concept that focuses on the overall health level of students' physical, psychological, and social adaptation, which determines that the public physical education curriculum should focus on the formation of students' lifelong physical exercise consciousness. It should strengthen the lifelong contact and interaction between sports and health, reshape students' sports behavior, make "martial arts" an indispensable part of life now and in the future, and realize the integration of martial arts and life, so that modern college students can calmly cope with the complex social life in terms of values, physical health, life ability, and work ability and can make continuous progress and development.

(10) Teaching sites and equipment of public physical education in colleges and universities are the hardware conditions for physical education teaching and the important guarantee for improving teaching quality. At present, due to the continuous expansion of college entrance examination and the increasing number of students in universities, there is a serious shortage of sports venues and equipment, and the problems of sports teaching venues and equipment are even more serious. Therefore, in order to ensure the quality of teaching, necessary measures should be taken to expand the public sports teaching venues and equipment in colleges and universities, so as to meet the requirements of students for physical education to the greatest extent. At present, there are three methods and measures as follows:

The first is to build sports venues and buy sports equipment. The most direct and effective way to change the current lack of public physical education teaching venues and equipment in colleges and universities is to build venues and purchase equipment. However, this method requires a lot of money, so not all schools can achieve and complete it. Therefore, the construction of venues and the purchase of equipment should be based on the acceptance and commitment of school funds and try to attract the support and sponsorship of social funds.

The second is to transform the original site and equipment. It is an important method and measure to reform the original venues and equipment to make up for the lack of funds. At present, every university that offers public physical education has 
some venues and equipment. Due to some reasons, these venues and equipment are unreasonable and out of repair. Therefore, we should strengthen the management and maintenance of the existing venues and equipment, ensure their use efficiency, and achieve the maximum utilization of them.

The third is to strengthen cooperation with relevant units and make use of existing sites and equipment of other units. At present, almost all colleges and universities are built in cities, which have certain martial arts venues and equipment. In the process of expanding the teaching sites and equipment of public physical education in colleges and universities, we should pay full attention to these resources, strengthen the cooperation with these units, and make use of the existing sites and equipment of other units. This process requires the relevant departments of the school to strengthen the contact and communication with other units and make full use of the venues and equipment of these units on the basis of scientific negotiation and equal transaction, applying the principle of doing the biggest thing with the least money.

(11) The construction of the soft environment of sports public curriculum should be strengthened. The soft environment refers to college students' understanding of sports, their love and pursuit of sports, their recognition and acceptance of the role of sports, and the sports tradition and atmosphere formed. Soft environment is a long-term forming process, which is also very important to the development of things. For physical education, a good soft environment can provide a good source for students and atmosphere support for public physical education in colleges and universities and promote the smooth development of public physical education. Therefore, measures should be taken to create and improve the soft environment of public curriculums in colleges and universities. At present, some measures can be taken, such as requiring well-known sports experts to give lectures, holding a series of high-level sports competitions, and vigorously publicizing and popularizing the basic knowledge and efficacy of sports. In the process of building and improving the soft environment of public physical education in colleges and universities, we should remember to be eager for quick success and instant benefit, blindly pursue speed and effect, and adhere to the guiding ideology of step by step.

\section{Conclusion}

The teaching evaluation index system of public physical education curriculum based on management by objectives is composed of 2 first-level indexes, 9 second-level indexes, and 22 third-level indexes, and the weight of each index is determined, which provides a relatively feasible standard for evaluating the teaching quality of public physical education curriculum. The evaluation index system of teaching quality of public physical education is a process in which various evaluation modes complement and blend with each other, and it will continue to change with the development of the times.

AHP involves human judgment in pairwise comparison of the order pairs, which makes the comparison biased. We tried to remove bias and make the judgment consistent, but still, due to leaning nature of human in favor of some alternative, the judgments are not completely pure. Current implementation in AHP could be expanded to other indexes and other qualitative/quantitative criteria. Techniques other than AHP, e.g., Fuzzy AHP, could be used, and results are compared.

The implementation of management by objectives is closely related to all departments and members of the school. It is necessary to encourage teachers and students to participate in the policy-making, evaluation, feedback, and other aspects of management by objectives. The objective evaluation results should be treated objectively and fairly, and an appropriate reward and punishment mechanism should be established to ensure the good effect of management by objectives and promote further improvement of teaching quality.

The purpose of this paper is to provide a new idea and comprehensive evaluation method for the evaluation of public physical education and to provide strategies for the teaching reform of public physical education. As an important means of monitoring physical education teaching, physical education teaching evaluation still needs continuous research and improvement.

\section{Data Availability}

The data used to support the findings of this study are available from the corresponding author upon request.

\section{Conflicts of Interest}

The authors declare that they have no conflicts of interest.

\section{Acknowledgments}

The study was supported by Science and Technology Project of China Railway Corporation, China (Grant no. 1341324011).

\section{References}

[1] E. Mccammon, A. Moore, C. P. Tyler et al., "6. Clinic quest: a game and curriculum to teach adolescents about the prevention and treatment of sexually transmitted infections," Journal of Adolescent Health, vol. 66, no. 2, pp. S3-S4, 2020.

[2] N. Siparsky, A. Wojtowicz, B. R. Veenstra, A. Madrigrano, and J. M. Velasco, "Novel simulation curriculum to teach surgical drain management," Journal of the American College of Surgeons, vol. 227, no. 4, p. e209, 2018.

[3] A. Ranauta, A. R. Tappuni, and P. Coulthard, "HIV teaching: a dental curriculum which fosters knowledge and attitude," Oral Diseases, vol. 26, no. S1, pp. 123-126, 2020. 
[4] S. Yu, "Evaluation of teaching quality based on fuzzy analytic hierarchy process," Computer Simulation, vol. 29, no. 6, pp. 369-372, 2019.

[5] M. E. Bernson-Leung and D. K. Urion, "The child neurology trainee-as-teacher: a clinical teaching curriculum tailored to learners' needs and developmental roles," Pediatric Neurology, vol. 78, no. 45, pp. 41-45, 2018.

[6] A. Almutlaq, M. Alshurem, M. Levesque-Roy, and R. Massie, "Teaching neuroimages: Sneddon syndrome," Neurology, vol. 93, no. 12, pp. e1227-e1228, 2019.

[7] E. Hornecker and M. Honauer, "Creating performance-oriented multimedia projects as part of an interdisciplinary teaching practice," IEEE Multimedia, vol. 25, no. 2, pp. 93-103, 2018.

[8] O. M. Steiner, "Chemical education: teaching science: a game for robotics?," Chimia, vol. 72, no. 7-8, p. 549, 2018.

[9] R. Kassa, F. Raslau, C. Smith, and P. Sudhakar, "Teaching neuroimages: leber hereditary optic neuropathy masquerading as neuromyelitis optica," Neurology, vol. 90, no. 1, pp. e94-e95, 2018.

[10] Y. Banu, A. Egemen, N. Sinan, and K. Murat, "Estimating suspended sediment load with multivariate adaptive regression spline, teaching-learning based optimization, and artificial bee colony models," Science of the Total Environment, vol. 639, no. 15, pp. 826-840, 2018.

[11] X. Yang, G. Lin, Y. Liu, F. Nie, and L. Lin, "Fast spectral embedded clustering based on structured graph learning for large-scale hyperspectral image," IEEE Geoscience and Remote Sensing Letters, vol. 5, pp. 1-5, 2020.

[12] S. A. Azer, "Race and culture in teaching cases," Academic Medicine, vol. 95, no. 2, pp. 173-174, 2020.

[13] P. Adam, C. F. Murphy, M. Dierich, and K. D. Hager, "Seven years of teaching communication with the patient-centered observation form," Family Medicine, vol. 50, no. 2, pp. 132-137, 2018.

[14] A. C. Knobloch, C. J. W. Ledford, S. Wilkes, and A. K. Saperstein, "The impact of near-peer teaching on medical students' transition to clerkships," Family Medicine, vol. 50, no. 1 , pp. 58-62, 2018.

[15] C. R. Shi, J. Rana, and S. Burgin, "Teaching \& learning tips 6: the flipped classroom," International Journal of Dermatology, vol. 57, no. 4, pp. 463-466, 2018.

[16] A. Aluko, J. Rana, and S. Burgin, "Teaching \& learning tips 8: preparing to teach in ambulatory settings," International Journal of Dermatology, vol. 57, no. 6, pp. 715-718, 2018.

[17] L. Zheng, C. Chen, W. Liu et al., "Enhancement of teaching outcome through neural prediction of the students' knowledge state," Human Brain Mapping, vol. 39, no. 2, pp. 3046-3057, 2018.

[18] J. B. Neal, R. A. Pearlman, D. B. White et al., "Policies for mandatory ethics consultations at U.S. academic teaching hospitals: a multisite survey study," Critical Care Medicine, vol. 48 , no. 6, pp. 847-853, 2020.

[19] A. Jaidka, D. Durocher, and P. Antiperovitch, "Cardioguide: a novel competency-based continuing professional development curriculum for cardiology," Canadian Journal of Cardiology, vol. 36, no. 10, pp. S39-S40, 2020.

[20] J. Gamo, "Assessing a virtual laboratory in optics as a complement to on-site teaching," IEEE Transactions on Education, vol. 62, no. 2, pp. 119-126, 2019. 